\title{
Reducing cost: What do hospitals gain from hiring clinical students?
}

\author{
Shari M. Burns ${ }^{* 1}$, Jacquelyn M. Smith², Thomas Virden III \\ ${ }^{1}$ Nurse Anesthesia Program, Midwestern University, Glendale, United States \\ ${ }^{2}$ College of Health Sciences-Glendale, Midwestern University, Glendale, United States \\ ${ }^{3}$ Clinical Psychology Program, Midwestern University, Glendale, United States
}

Received: January 18, 2015

DOI: $10.5430 /$ jnep.v5n5p82
Accepted: February 26, $2015 \quad$ Online Published: March 13, 2015

URL: http://dx.doi.org/10.5430/jnep.v5n5p82

\begin{abstract}
Objective: The purpose of this retrospective descriptive correlational study was to 1) identify factors that played a role in hiring decisions for student nurse anesthetists following graduation; and 2) to identify the extent and nature of benefits afforded clinical sites who train student nurse anesthetists.

Methods: An on-line survey was used to collect data regarding hiring decisions including the nature of benefits. The survey examined hiring practices for clinical sites where 118 student nurse anesthetists who graduated from the Nurse Anesthetist Program at Midwestern University (2006-2013) received clinical education. The anonymous on-line survey was sent to 45 chief Certified Registered Nurse Anesthetists (CRNAs), clinical coordinators or anesthesia department chairpersons at 25 clinical facilities who were involved in hiring.

Results: Multiple factors were identified as playing a role in hiring CRNAs who trained at the institution. Key factors included interpersonal skills; ability to fill a key position at a rural healthcare facility; and willingness to contribute to the healthcare team. Hiring CRNAs who performed clinical rotations reduced orientation time and supervision requirements.

Conclusions: Identifying factors that influence hiring practices for CRNAs following graduation is important to the graduate and the institution. Informing hospitals regarding the tangible benefits of hiring CRNAs who gain clinical experience at their institutions is vital. This effort may open the door for additional clinical sites interested in teaching future healthcare providers. Given soaring healthcare costs, follow-on studies are needed to explore hiring practices across healthcare disciplines. Specifically, examining the relationship between clinical education and institutional gains may benefit all stakeholders.
\end{abstract}

Key Words: Healthcare hiring, Clinical education, Hiring practices

\section{INTRODUCTION}

Throughout allied health clinical education, students hone skills and apply knowledge essential for practice regardless of professional discipline. Frequently, graduates are hired at clinical sites where they previously rotated. This practice led to the perception that clinical rotations were beneficial to clinical sites as well as students. In order to quantify this perception, a descriptive correlational study was conducted.
The study provided foundation for future studies on this important topic. The purpose of this retrospective study was to: 1) identify factors that played a role in hiring student nurse anesthetists following graduation; and 2) to identify the extent and nature of benefits afforded clinical sites who train student nurse anesthetists.

Results from this study offer information to hospitals regarding the benefits of hiring students who perform clinical

*Correspondence: Shari M. Burns; Email: sburns@midwestern.edu; Address: Nurse Anesthesia Program, Midwestern University, Glendale, AZ19555 N 59th Ave Glendale, United States. 
rotations at specific sites. These tangible benefits may encourage hospitals to consider professional clinical education training programs and lead to greater opportunities for clinical education for student nurse anesthetists and other health professions.

Multiple studies from a variety of professions, including a study conducted by nurse anesthetists, have examined the factors that influence hiring of health professional students. Personality traits, professional behavior and communication have been important considerations when hiring new graduates into non-health as well as health care professions. For example, Green, Graybeal and Madison (2011) asked accounting students to rank in importance personality, technical skills, and interpersonal skills before and after an accounting internship. ${ }^{[1]}$ Employers ranked the same traits and the rankings of students were compared to the employer's rankings. Overall, students valued personality and interpersonal traits more than technical and traits. Employers consistently ranked personality traits as more important than did either pre- or post-internship students. In addition, work ethic remained a highly ranked trait by employers as compared to post-internship students. Professional exposure during the internship however, shifted the student's perception of professional behavior closer to that of the employer. The study emphasized the need for educators to consider the value of an accounting internship in preparing students for the workplace. Communication, professional behavior and personality traits are also important hiring considerations for graduates of health care professions.

McCauley, Meixner, Harwell and Onsomu (2011) studied clinical laboratory practitioners and educators and reported that student affective competencies scored highest when examining clinical preparedness of students for practice. ${ }^{[2]}$ Student affective competency includes professional conduct, demonstrating safe laboratory practices as well as adhering to policies and procedures. Practitioners also agreed that cognitive and psychomotor skills were strongly linked to educational preparedness for clinical practice. The study noted that strong communication between clinical practitioners and educators was vital to laboratory science market needs. In a similar study of pharmacy graduates strong communication and professional behavior were highly regarded by pharmacists when hiring pharmacy graduates. ${ }^{[3]}$

Studies have evaluated possible benefits to the institution when a graduate is hired that had completed a clinical experience at that institution. In particular, it has been proposed that this practice may help to address the deleterious consequences of the staffing shortages. For example, as part of a nursing co-op program, a pediatric nephrology unit hired a nursing student so the student could learn multiple aspects of the specialty. ${ }^{[4]}$ As a result of this clinical training in the specialty, the authors concluded that new graduates that had completed part of undergraduate clinical work in the specialty setting were viable candidates for employment in the specialty; traditionally, specialties were regarded as positions for experienced nurses. Ultimately, these results suggested a mechanism to address the nursing shortage in the specialty which benefitted the hospital. In a separate study, Coackley and Ghiloni (2009) examined the benefits of a fellowship program providing undergraduate nursing students the opportunity to actively learn oncology nursing initiated at the Massachusetts General Hospital. ${ }^{[5]}$ Following execution of the oncology nursing fellowship, a qualitative study was conducted using focus groups. The purpose of the study was to determine how the fellowship influenced the student's perception of preparation for role as an oncology nurse. Two themes emerged: the fellowship provided first-hand experience with preceptor role modeling and secondly, the fellowship facilitated relationship building with staff, patients and families. More importantly, relationships with staff positively influenced the decision to return to the clinical site for employment.

In a similar approach, the benefits of a partnership model between a large California State University School of Nursing and Long Beach Memorial Medical Center was examined by Huckabay (2009). The purposes of the partnership were twofold: 1) to improve the supply of nurses in light of impending retirements that would result in a significant nursing shortage for the hospital; and 2) to solve the problem of a struggling nursing program based on state budget cuts. Results of the study revealed significant value of the partnership to both entities. Ninety-five percent of the nursing graduates were hired, illustrating a solid gain for healthcare agencies who educate nursing students. ${ }^{[6]}$ The nursing program regained viability and the hospital hired nurses trained at their facility.

Finally, in a study that evaluated employment of graduate Certified Registered Nurse Anesthetists (CRNAs) over a 12 year period, Wachtel and Dexter $(2012)^{[7]}$ reported that the graduates were more likely to work and be hired at rotationbased hospitals although no information was collected from the employers about specific factors that had influenced their hiring practices.

For the current study, it is useful to know that the Midwestern University Nurse Anesthesia students rotate to clinical sites, in and outside of Arizona where the campus is located. The five rotations span 15 months (5 quarters) following one year of didactic education. Students rotate to individual clinical sites 4-5 times during the 15 months depending on required 
specialty cases (obstetric, pediatric, neurosurgery, cardiac) involved in hiring CRNAs at 25 clinical facilities.

as well as overall numbers of anesthetic cases.

\section{METHOD}

\subsection{Design, sample and ethical considerations}

Following Institutional Review Board approval, a retrospective descriptive correlational study was used to examine hiring practices for clinical sites where 118 student nurse anesthetists who graduated from the Nurse Anesthetist Program at Midwestern University from 2006 ( $1^{\text {st }}$ program cohort) through 2013 received clinical education. The anonymous on-line survey was sent to 45 Chief CRNAs, Clinical Coordinators or anesthesia department chairpersons who were

\subsection{Instrumentation}

A seven (7)-item online survey was generated to gather data (see Table 1). Survey content focused on hiring practices in anesthesia departments. Specifically, the survey addressed tangible as well as intangible benefits to anesthesia departments and institutions. Using an expert in survey construction, the survey was examined for content validity. In addition, the survey was sent to five (5) nurse anesthetists involved in hiring practices to assure items were understandable (reliable). All five nurse anesthetists agreed that the survey items were reliable and valid. The nurse anesthetists were not included in the study sample.

Table 1. Survey

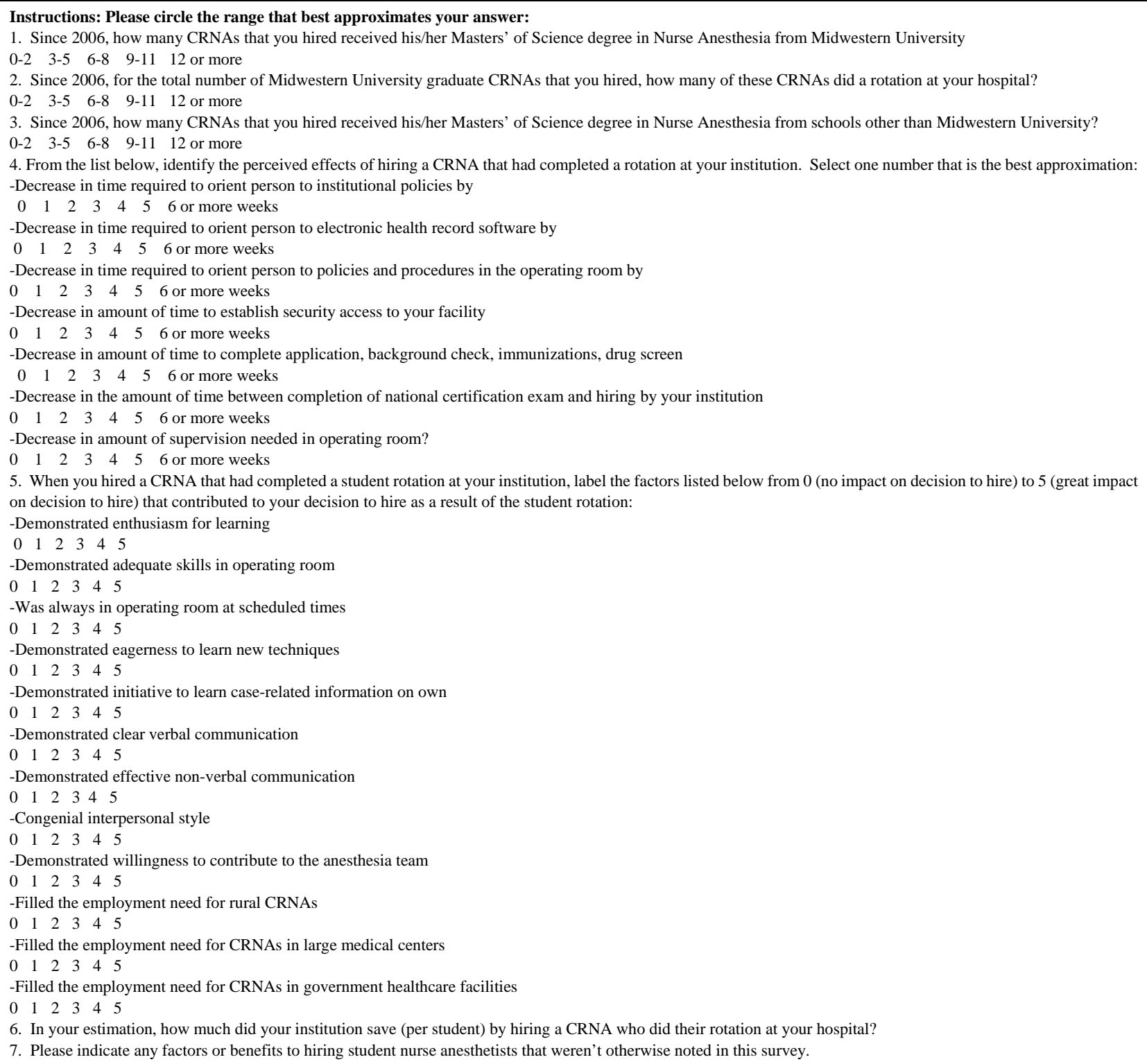




\subsection{Procedures}

The anonymous on-line survey was sent to 45 Chief CRNAs, Clinical Coordinators or anesthesia department chairpersons at 25 clinical facilities. At some clinical sites, multiple coordinators exist. To gain the greatest survey return rate, the researchers sent a pre-notification two weeks prior to emailing the survey link. Once the link for the survey was emailed, a second request for participation was sent two weeks later. Data was securely stored in the Nurse Anesthesia Program office, Midwestern University Glendale, Arizona.

\subsection{Data analysis}

Descriptive statistics were used to summarize the data. Pearson's correlation coefficient was used to identify relationships between responses to questions regarding time saved training new CRNA as well as impact of variables on the decision to hire.

\section{RESUlts}

Thirty one percent (14/45) of the surveys were returned from individuals at 25 clinical sites. Combining all the sites, between 1 and 2 Midwestern University (MWU) graduates were hired by the surveyed institutions (average $1.57 \pm 0.39$ ) compared to 2-3 graduates from non-MWU institutions (average $2.14 \pm 0.30$ ) institutions. Between 1 and 2 Midwestern graduates that here hired by the institution had also completed a rotation at the institution (average $1.57 \pm 0.39$ ). All of the MWU graduates that were hired by the institutions had completed a rotation at the site.

Factors including enthusiasm for learning, clinical skills, timeliness, eagerness to learn new techniques, initiative to learn case-related information on own, congeniality, willingness to contribute to the team and both verbal and non-verbal communication had a similar positive impact on the decision to hire the graduate CRNA (see Table 2). In contrast, the staffing needs of a particular type of site had minimal impact on the decision to hire (see Table 2).

Hiring of MWU graduates who had previously completed a rotation at the institution decreased the amount of time needed for supervision in the operating room by 1-2 weeks (see Table 3, $1.29 \pm 0.22$ ). Completion of prior rotation at the institution also decreased by 1-2 weeks the time required to orient the person to institutional policies, to orient to electronic health records, to orient to policies and procedures in the operating room, to establish security access to the facility, to complete background check/immunizations, and time between completion of certification exam and hiring (see Table $3)$.

Table 2. Impact of factors from 0 (no impact) to 5 (great impact) on decision to hire graduate CRNA

\begin{tabular}{ll}
\hline & Average \pm SE \\
\hline Demonstrated enthusiasm for learning & $3.21 \pm 0.47$ \\
Demonstrated adequate skills in operating room & $3.29 \pm 0.49$ \\
Was always in operating room at scheduled times & $3.21 \pm 0.47$ \\
Demonstrated eagerness to learn new techniques & $3.29 \pm 0.49$ \\
Demonstrated initiative to learn case-related information on own & $3.29 \pm 0.49$ \\
Demonstrated clear verbal communication & $3.21 \pm 0.48$ \\
Demonstrated effective non-verbal communication & $3.13 \pm 0.47$ \\
Congenial interpersonal style & $3.21 \pm 0.45$ \\
Demonstrated willingness to contribute to the anesthesia team & $3.43 \pm 0.49$ \\
Filled the employment need for rural CRNA & $1.21 \pm 0.56$ \\
Filled the employment need for CRNA in large medical center & $1.36 \pm 0.44$ \\
Filled employment need for CRNA in government healthcare facility & $0.71 \pm 0.34$ \\
\hline
\end{tabular}

Table 3. Perceived decrease in weeks required for completion of the following items when graduate CRNA were hired that had completed rotation at the institution

\begin{tabular}{ll}
\hline & Average \pm SE \\
\hline Decrease in time required to orient person to institutional policies & $1.20 \pm 0.22$ \\
Decrease in time required to orient person to electronic health record software & $1.14 \pm 0.27$ \\
Decrease in time required to orient person to policies and procedures in operating room & $1.07 \pm 0.22$ \\
Decrease in time amount of time to establish security access to the facility & $0.79 \pm 0.21$ \\
Decrease in amount of time to complete application, background check, immunizations, drug screen & $1.36 \pm 0.49$ \\
Decrease in amount of time between completion of certification exam and hiring by institution & $1.14 \pm 0.40$ \\
Decrease in amount of supervision needed in operating room & $1.29 \pm 0.34$ \\
\hline
\end{tabular}


Positive correlations were noted between the amount of supervision needed in the operating room and time required to orient the CRNA to institutional policies $(r=0.730, \mathrm{n}=15$, $p=.002)$; electronic health record software $(r=0.903, \mathrm{n}=$ $15, p<.001)$; operating room policies $(r=0.838, \mathrm{n}=15, p$ $<.001)$ and establishing security access to the facility $(r=$ 0.689, $\mathrm{n}=15, p=.005)$.

Positive correlations were also found between the amount of supervision needed in the operating room and the amount of time needed to complete application, background check, immunizations, and drug screens $(r=0.701, \mathrm{n}=15, p=.004)$ and between completion of national certification exam and hiring by the institution ( $r=0.707, \mathrm{n}=15, p=.003$ ). Only two sets of correlations were performed. Significant negative correlations were absent in the study.

The participants estimated that the institution saved $\$ 2,000$ $\$ 3,000$ by hiring a CRNA who completed clinical education at the hospital. No additional factors or benefits to hiring student nurse anesthetists were noted by those who filled out the survey.

\section{Discussion}

Professional clinical education offers training hospitals the opportunity to recruit prospective employees. Preceptors have the opportunity to preview the personality traits, communication skills and technical practice skills of students that complete clinical education at the healthcare institution. The current study suggests additional tangible and intangible benefits of employing CRNAs who completed clinical education at the facilities. Hiring CRNAs who performed clinical rotations at the facilities reduced orientation at least one or more weeks. In today's healthcare environment, reduction of orientation time translates into significant savings to departments and hospitals as healthcare facilities continue to create ways to minimize costs. While it is not possible to offer conclusions of cost savings, the current study shared that savings approximated $\$ 2,000.00-\$ 3,000.00$ for CRNAs who had trained at the site prior to hiring. The new graduates required less time in orientation activities overall, but exact times for each activity (institutional policies, operating room policies and electronic record).

Enhanced professionalism is a byproduct of clinical education that aligns with healthcare institutional expectations and influences hiring practices. The current study revealed significant relationships between time for orientation (addressing operating room policies, securing access to the facility, electronic health record policies) and interpersonal variables (enthusiasm, communication skills, timeliness, congeniality).
The authors chose to examine these relationships to add richness to the study. Perhaps these traits also influenced hiring the new graduates. These results are consistent with the findings that of emphasized professionalism and communication skills as pivotal for hiring practices. ${ }^{[1,3]}$

Similar to the findings in the current study, Henderson (2012) asserted that student clinical experience serves as a viable resource for future employment. ${ }^{[8]}$ In his study, Henderson reported than an increased minority workforce was attractive to future employers and, in an effort to foster entry of minorities into healthcare careers, the benefits of internships for minority students was examined. The authors conveyed that an increased minority workforce was attractive to future employers. However, no data was offered regarding the hiring of minorities at clinical sites following their internships.

Further research is needed to determine retention rates at healthcare facilities where nurse anesthesia training took place. Again, the cost analysis may offer meaningful data for facilities hiring practices. Hillman (2011) examined retention of nursing staff following a new graduate transition programme and found major cost saving to organizations. Similarly, previous clinical education may afford institutions long-term cost savings. ${ }^{[9]}$

\section{Limitations}

Limitations exist for the current study. Data was self-reported by anesthesia department personnel. It would have been advantageous to retrieve additional information from the Human Resources department. However, in some facilities, Certified Registered Nurse Anesthetists are independent practitioners that contract with anesthesia groups as opposed to employment by the hospital. While the sample size is adequate, a larger sample size may have yielded more meaningful results. In addition, the study focused on graduates from one nurse anesthesia program, but also inquired about new hires from other nurse anesthesia programs. Because clinical sites were surveyed in limited geographical areas, it is difficult to generalize the findings. Since the survey data was anonymous, it is not possible to determine how many individual reported from the same institution. In addition, the study did not determine who did the hiring.

\section{Conclusion}

The current study serves as a springboard for additional research. This effort may open the door for additional clinical sites interested in teaching future healthcare providers. A multi-program study as well as interdisciplinary study may yield valuable results that further inform hospitals as to the tangible benefits of hiring healthcare professionals that ro- 
tate at the clinical sites. For example, examining the hiring of physician assistants or nurse practitioners who rotate at clinical sites may offer additional information that benefit employers. Further research is needed to determine if studies similar to the current study increase the number of hospitalbased clinical rotations. Finally, an actual cost study may positively influence the availability of clinical rotation sites for health professions.

\section{CONFliCtS OF INTEREST DisClOSURE}

The authors declare that there is no conflict of interest statement.

\section{REFERENCES}

[1] Green BP, Graybeal P, Madison RL. An exploratory study of the effect of professional internships on students' perception of the importance of employment traits. Journal of Education for Business. 2011; 86(2): 100. http://dx.doi.org/10.1080/08832323.2010.480992

[2] McCauley G, Meixner J, Harwell G, et al. Preparation for Clinical Laboratory Practice: A Practitioners' Point of View to Enhance Students' Experiences and Workforce Needs. Res, Educ and Policy. 2011; 4(2): 669-680.

[3] Thompson D, Nuffer W, Brown K. Characteristics valued by the pharmacy practice community when hiring a recently graduated pharmacist. American Journal of Pharmaceutical Education [serial online]. 2012; 76(9): 1-8. PMid:23193334 http://dx.doi.org/10.5688 /ajpe769170

[4] Windt K, Breen C, McNellis L, et al. Recruitment and retention in pediatric nephrology nursing: Reaching out at the student level. Nephrology Nursing Journal. 2008; 35(2): 182.

[5] Coakley AA. A fellowship program preparing students for employment as new graduate nurses in oncology nursing. Creative Nursing.
2009; 15(1): 46. http://dx.doi.org/10.1891/1078-4535.15 .1 .46

[6] Huckabay LM. Partnership between an educational institution and a healthcare agency-Lessons learned: Part I. Nursing Forum. 2009; 44(3): 154-164. PMid:19691651 http://dx.doi.org/10.1111 /j.1744-6198.2009.00138.x

[7] Wachtel RE, Dexter F. Training rotations at hospitals as a recruitment tool for Certified Registered Nurse Anesthetists. AANA Journal. 2012; 80(4): S45-48.

[8] Henderson D, Jackson V, Simmons S, et al. Preparation for successful clinical experiences: A collaborative perspective on internship orientation. Journal of Best Practices in Health Professions Diversity: Education, Research \& Policy. 2012; 5(1): 774-789. PMid:23248830

[9] Hillmann L, Foster RR. The impact of a nursing transitions programme on retention and cost savings. Journal of Nursing Management. 2011: 19(1): 50-56. PMid:21223405 http://dx.doi.org /10.1111/j.1365-2834.2010.01187.x 